\title{
Significance of "Not Detected but Amplified" Results by Real-Time PCR Method for HPV DNA Detection
}

\author{
Taek Soo Kim, ${ }^{1}$ Mi Suk Lim, ${ }^{2}$ Yun Ji Hong, ${ }^{2}$ Sang Mee Hwang, ${ }^{2}$ Kyoung Un Park, ${ }^{2,3}$ \\ Junghan Song, ${ }^{2,3}$ and Eui-Chong Kim ${ }^{1,3}$ \\ ${ }^{1}$ Department of Laboratory Medicine, Seoul National University Hospital, 101 Daehak-ro, Jongno-gu, Seoul 03080, Republic of Korea \\ ${ }^{2}$ Department of Laboratory Medicine, Seoul National University Bundang Hospital, 82 Gumi-ro, 173 Beon-gil, Bundang-gu, \\ Seongnam-si, Gyeonggi-do 13620, Republic of Korea \\ ${ }^{3}$ Department of Laboratory Medicine, Seoul National University College of Medicine, 101 Daehak-ro, Jongno-gu, \\ Seoul 03080, Republic of Korea
}

Correspondence should be addressed to Kyoung Un Park; m91w95pf@snu.ac.kr

Received 24 March 2016; Accepted 21 November 2016

Academic Editor: Patrizia Cardelli

Copyright (C) 2016 Taek Soo Kim et al. This is an open access article distributed under the Creative Commons Attribution License, which permits unrestricted use, distribution, and reproduction in any medium, provided the original work is properly cited.

\begin{abstract}
Human papillomavirus (HPV) infection is an important etiologic factor in cervical carcinogenesis. Various HPV DNA detection methods have been evaluated for clinicopathological level. For the specimens with normal cytological finding, discrepancies among the detection methods were frequently found and adequate interpretation can be difficult. 6,322 clinical specimens were submitted and evaluated for real-time PCR and Hybrid Capture 2 (HC2). 573 positive or "Not Detected but Amplified" (NDBA) specimens by real-time PCR were additionally tested using genetic analyzer. For the reliability of real-time PCR, 325 retests were performed. Optimal cut-off cycle threshold $\left(C_{T}\right)$ value was evaluated also. $78.7 \%$ of submitted specimens showed normal or nonspecific cytological finding. The distributions of HPV types by real-time PCR were not different between positive and NDBA cases. For positive cases by fragment analysis, concordance rates with real-time PCR and HC2 were 94.2\% and 84.2\%. In NDBA cases, fragment analysis and real-time PCR showed identical results in $77.0 \%$ and HC2 revealed $27.6 \%$ of concordance with fragment analysis. Optimal cut-off $C_{T}$ value was different for HPV types. NDBA results in real-time PCR should be regarded as equivocal, not negative. The adjustment of cut-off $C_{T}$ value for HPV types will be helpful for the appropriate result interpretation.
\end{abstract}

\section{Introduction}

Persistent infection with one or more carcinogenic types of human papillomavirus (HPV) is an important etiologic factor in the development of cervical intraepithelial neoplasia and the progression to cervical cancer [1-3], the third common cause of cancer mortality in women worldwide [4]. HPV infection causes virtually all cases of cervical cancer and a less-defined, smaller fraction of vaginal, vulvar, penile, and anal cancers. Moreover, cervical infection with high risk HPV is associated with preterm birth and placental abnormalities in pregnant women [5]. Cytopathology has provoked the marked reduction of cervical cancer mortality, but its sensitivity is actually lower than that of HPV DNA assays [6]. Based upon this agreement, some researchers insisted that the screening interval could be extended to 6 years (10 years for women aged 50 and over) in HPV testing replaced cytology as the primary screening test [7].

Until now, more than $100 \mathrm{HPV}$ types have been identified and fully sequenced [8]. Approximately $40 \mathrm{HPV}$ types infect the anogenital tract and fifteen HPV types, $16,18,31,33,35$, 39, $45,51,52,56,58,59,68,73$, and 82 , are considered as highly oncogenic (high risk HPV) and HPV types 26,53 , and 66 as probably oncogenic, while HPV types $6,11,40,42,43,44,54$, $61,70,72,81$, and CP6108 are classified as viruses with low oncogenic potential (low risk HPV) [9]. As well as in nearly all abnormal cytology samples, high risk HPV DNA has been detected in a high percent of cytological "negative for intraepithelial lesion or malignancy (NILM)" samples [10, 11]. In other words, HPV is known to be detectable in virtually all abnormal cervical samples; how about in NILM samples? So we evaluated "Not Detected but Amplified (NDBA)" results 
that could be low-copy of high risk HPV DNA and/or crossreaction with nonhigh risk HPV types, when using real-time PCR method compared with the results for other assays.

\section{Materials and Methods}

2.1. Study Population. From April 2010 to July 2012, 6,322 clinical specimens were submitted for HPV DNA test at Seoul National University Bundang Hospital. 814 specimens showing positive and NDBA results by real-time PCR method were evaluated in this study.

2.2. Papanicolaou (Pap) Tests. All women were first subjected to a conventional cervicovaginal Pap smear. Pap smear abnormalities were interpreted and classified by using the 2001 Bethesda System [12]. An additional sample for the detection of HPV DNA was taken from the cervix by using the sampling kit for the Hybrid Capture 2 (HC2; Qiagen, Hilden, Germany).

2.3. HPV Detection by Real-Time PCR. The Abbott RealTime High Risk HPV test (Abbott, Wiesbaden, Germany) was performed with the fully automated nucleic acid preparation instrument $m 2000$ sp (Abbott) and the real-time PCR instrument m2000rt (Abbott) by following the manufacturer's instructions as previously described [13]. The assay uses four channels for the detection of fluorescent signals: one for the detection of an internal control (136-bp region of human $\beta$ globin), a second one for the detection of HPV 16, a third one for the detection of HPV 18, and a fourth one for the detection of the high risk common $12 \mathrm{HPV}$ types, that is, 31 , $33,35,39,45,51,52,56,58,59,66$, and 68. PCR amplification of HPV targets was achieved using a modified GP5+/6+ primer mixture consisting of three forward and two reverse primers. The assay cut-off is set at a fixed cycle threshold $\left(C_{T}\right)$ value of 32, which is already established by the manufacturer. On the interpretation of amplification curve, amplification above the target $C_{T}$ value refers to new term "Not Detected but Amplified (NDBA)." For 325 of 814 specimens showing NDBA or positive results, the specimens were refrigerated at $4^{\circ} \mathrm{C}$. After 2 or 4 days, DNA extraction and real-time PCR were repeated by the same technologist.

2.4. HR HPV Detection by HC2 Assay. HC2 test was also performed on the Digene Specimen Transport Medium (STM; Qiagen, Hilden, Germany) specimen throughout the study in accordance with the manufacturer's instructions and as previously described [14]. Specimens are stored in the STM tubes at $4^{\circ} \mathrm{C}$ until use. The hybridization RNA probes used were directed against high risk HPV types 16, 18, 31, 33, 35, 39, $45,51,52,56,58,59$, and 68 , as described by the manufacturer. Samples were classified as high risk HPV DNA positive if the relative light unit/cut-off (RLU/CO) ratio reading obtained from the luminometer was 1.0 or greater.

2.5. HPV Detection Using Genetic Analyzer. Using 3130xl genetic analyzer (Applied Biosystems, Foster, USA), fragment analysis were performed. To detect the HPV type(s) present in a sample, the samples showing positive and NDBA results by real-time PCR were tested additionally and this method is capable of recognizing 18 different HPV types including 13 high risk $(16,18,31,33,35,39,45,51,52,56,58,59$, and 68) and 5 low risk types. DNA was extracted using QIAamp viral RNA kit (Qiagen, Hilden, Germany) and amplified through 40 cycles consisting of $30 \mathrm{sec}$ at $95^{\circ} \mathrm{C}, 90 \mathrm{sec}$ at $60^{\circ} \mathrm{C}$, and $90 \mathrm{sec}$ at $72^{\circ} \mathrm{C}$. PCR products were purified with $1 \mu \mathrm{L}$ of shrimp alkaline phosphatase (SAP) under the condition of $35 \mathrm{~min}$ at $35^{\circ} \mathrm{C}$ followed by $15 \mathrm{~min}$ at $65^{\circ} \mathrm{C}$. Purified PCR products were analyzed with GeneMapper software version 4.0 (Applied Biosystems, Foster, USA).

2.6. Statistical Analysis. Statistical analysis of the data was performed and included the 2-tailed chi-square test for comparison of NDBA results and positive results in real-time PCR. The statistics were calculated using Analyse-it (version 2.30, Analyse-it Software, Ltd., Leeds, UK). Statistical significance was set at a level of $<0.05$.

\section{Results}

3.1. Specimen Demographics. Totally, 6,322 clinical specimens from health promotion center $(6,036,95.5 \%)$, the department of obstetrics and gynecology (272, 4.3\%), and other departments $(14,0.2 \%)$ were submitted at the Department of Laboratory Medicine, Seoul National University Bundang Hospital. Through the chart reviews, Pap smear results were reported as "negative for intraepithelial lesion or malignancy (NILM)" in 4516 (71.4\%) specimens, "reactive cellular change" in 117 (1.9\%) specimens, "reactive cellular changes associated with inflammation (includes typical repair)" in 347 (5.5\%) specimens, "high grade squamous intraepithelial lesion (HSIL)" in $7(0.1 \%)$ specimens, "low grade squamous intraepithelial lesion (LSIL)" in 26 (0.4\%) specimens, "atypical squamous cells of undetermined significance (ASC-US)" in $115(1.8 \%)$ specimens, "atrophy" in 251 (3.0\%) specimens, "chronic cervicitis" in $3(0.1 \%)$ specimens, and the descriptive reports not mentioned above in 749 (11.9\%) specimens. In 191 (3.0\%) specimens, Pap smear results were not reported or tests were not performed.

3.2. Concordance among Multiple Methods. Positive or NDBA results in real-time PCR were shown in 816 (12.9\%) specimens. For 763 (12.1\%) specimens, fragment analysis was performed and high risk HPV types were identified in 582 (9.2\%) specimens. Positivity for HC2 was shown in 544 (8.6\%) specimens and high risk HPV types for real-time PCR were identified in 593 (9.4\%) specimens. In 479 (7.6\%) specimens, HC2 and real-time PCR revealed concordant results (positive in HC2 and high risk HPV types in real-time PCR).

In $593(9.4 \%)$ and $221(3.5 \%)$ out of 6,322 specimens, positive and NDBA results for high risk HPV types by realtime PCR were obtained and described with mean $C_{T}$ value (Table 1). The distribution of high risk HPV type for positive and NDBA results are not significantly different $(p>0.05)$.

In NDBA results by real-time PCR, HC2 shows correspondence rate of $20.4 \%$ in HRC, $21.1 \%$ in type 16 , and $38.1 \%$ in type 18, respectively. For 14 mixed HPV type, HC2 revealed 
TABLE 1: Distribution of high risk HPV types for positive and NDBA results by real-time PCR.

\begin{tabular}{|c|c|c|c|c|c|c|c|}
\hline & Type 16 & Type 18 & HRC & Type 16 \& HRC & Type 18 \& HRC & Types 16 \& 18 & Total \\
\hline Positive & 55 & 33 & 482 & 12 & 10 & 1 & 593 \\
\hline$C_{T \_ \text {Mean }}$ & 25.1 & 26.5 & 25.6 & $25.0 / 26.9$ & $25.2 / 25.4$ & $24.1 / 23.2$ & \\
\hline NDBA & 19 & 21 & 167 & 9 & 5 & 0 & 221 \\
\hline$C_{T_{\text {_Mean }}}$ & 34.6 & 34.9 & 34.0 & $34.6 / 34.3$ & $35.5 / 34.0$ & - & \\
\hline
\end{tabular}

negativity. In positive results by real-time PCR, HC2 revealed the positivity in $82.0 \%$ (HRC), $72.7 \%$ (type 16 ), 66.7\% (type 18), and $95.7 \%$ (mixed type), respectively (Table 2 ).

For the results by fragment analysis, real-time PCR shows the detection rates of $79.7 \%$ (type 16), 92\% (type 18), 96.4\% (HRC), and 32.5\% (mixed type) including NDBA. HC2 detected 57.6\% (type 16), 68.0\% (type 18), 78.4\% (HRC), and $75.0 \%$ (mixed type) for types identified by fragmented analysis (Table 3). Overall, real-time PCR detects correctly 516 (90.1\%) of 573 fragments analysis results. On the other hand, HC2 detects 433 (75.6\%) of fragment analysis. For NDBA results identified as high risk HPV by fragment analysis, real-time PCR revealed $77.0 \%$ (67/87) of concordance rate, whereas HC2 showed 27.6\% (24/87) (Table 4).

3.3. Result Interpretation by Cycle Threshold $\left(C_{T}\right)$ Changes. Up to the change of cut-off $C_{T}$ from 31 to 34 , sensitivity and specificity of real-time PCR were described in Table 5. When drawing ROC decision plot, areas under the ROC curve (AUCs) were 0.86 for HPV type 16, 0.98 for HPV type 18 , and 0.76 for HRC. Optimal cut-off $C_{T}$ values were 35.58 in type $16,34.01$ in type 18 , and 31.99 in high risk common types.

3.4. Repeatability. For evaluation of repeatability, retests were performed in 325 specimens showing the presence of type 16 HPV DNA, type 18 HPV DNA, or HRC HPV DNA including NDBA results. For type 16 HPV DNA, retests were done in 53 specimens and amplification curve was observed in 43 (81.1\%) specimens. As shown in Figure 1, SD difference was 0.873 and upper and lower margins of 95\% limits of agreement were 1.384 and -2.037 , respectively. In 43 specimens showing amplification, 5 retest results showed $C_{T}$ value larger than 32. For 10 specimens, no amplification curve was observed at repetition, and they showed initial $C_{T}$ value larger than 32 . In case of type 18, retests were performed in 22 specimens and 15 specimens showed amplification at repetition. No cases revealed $C_{T}$ value larger than 32 and 7 cases did not show any amplification at repetition. The initial $C_{T}$ values in 7 cases were larger than 32 . In 15 specimens, SD difference was 0.842 and upper and lower margins of $95 \%$ limits of agreement were 1.558 and -1.744 , respectively.

In case of HRC HPV DNA, 265 specimens showed amplification at repetition and SD of difference was 1.3. The upper and lower margins of $95 \%$ limits of agreement were 2.352 and -2.744, respectively. In 17 specimens, no amplification was observed at repetition. For 10 specimens showing initial $C_{T}$ value larger than 32,3 specimens showed $C_{T}$ value under 32 at repetition. For 18 specimens larger than $32 C_{T}$ value at repetition, 11 specimens showed initial $C_{T}$ value under 32 . Ten of 17 specimens showing no amplification showed $C_{T}$ values larger than 32 at initial test.

\section{Discussion}

Differently from other previous evaluation studies, our study population was mainly limited to the specimens showing the normal or nonspecific cytological findings (NILM, reactive cellular change, atrophy, etc.). So, the cytological or pathologic finding was not helpful for the prediction of HPV existence in this study. HPV load and cumulative incidence of cervical lesion are known to be significantly correlated $[15,16]$. At the view of guideline change, the position of HPV DNA test moves from the adjunctive test method to cotest method. Although PCR has been the "gold standard" technique in HPV diagnostics, the disadvantages of PCR are its extremely high analytical sensitivity and potential for contamination, leading to false-positive results [14]. But, as revealed in NDBA results, real-time PCR results showing amplification curve above $C_{T}$ were needed to be reconsidered carefully. The accuracy of detection of high risk HPV is known to be significantly higher with Abbott RealTime High Risk HPV than HC2 [17]. HC2 technology measures sensitivity versus defined clinical endpoints (CIN 3+/SCC) and ensures reporting of positive results only when risk of disease progression exists. The limit of detection of HC2 is 5,000 copies/mL; it is much lower when compared to less than 10 copies of PCR [18].

Also, in results showing specific HPV types by fragment analysis, real-time PCR shows higher concordance rate than HC2. Particularly, in specimens showing NDBA, HC2 tends to reveal negative results much more frequently. Considering the distributions of HPV types in NDBA and positive results, the concordance rates between fragment analysis and realtime PCR, and the results of repeatability tests, NDBA results should be regarded as equivocal or positive, not as negative.

According to AUC value for the $C_{T}$ change, appropriate $C_{T}$ was different for HPV types. In HRC, $C_{T}$ of 32 is appropriate as described by manufacturer, but in types 16 and 18, $C_{T}$ of 33 or 34 will be more suitable.

In 167 results with negative result by $\mathrm{HC} 2$ and NDBA by real-time PCR, 58 (34.7\%) results were assigned to the specific high risk HPV types by fragment analysis. Out of 58 results, 52 (89.6\%) high risk HPV types were detected by real-time PCR and fragment analysis. Using HC2 only, false-negative results can be reported in specimens with low level persistent infection. The clinical relevance and implications of low level persistence of HPV are not clearly known, nor is the cause of low level persistence. In a previous study by Collins et al., integration of HPV 16 resulted in a markedly lower viral copy 


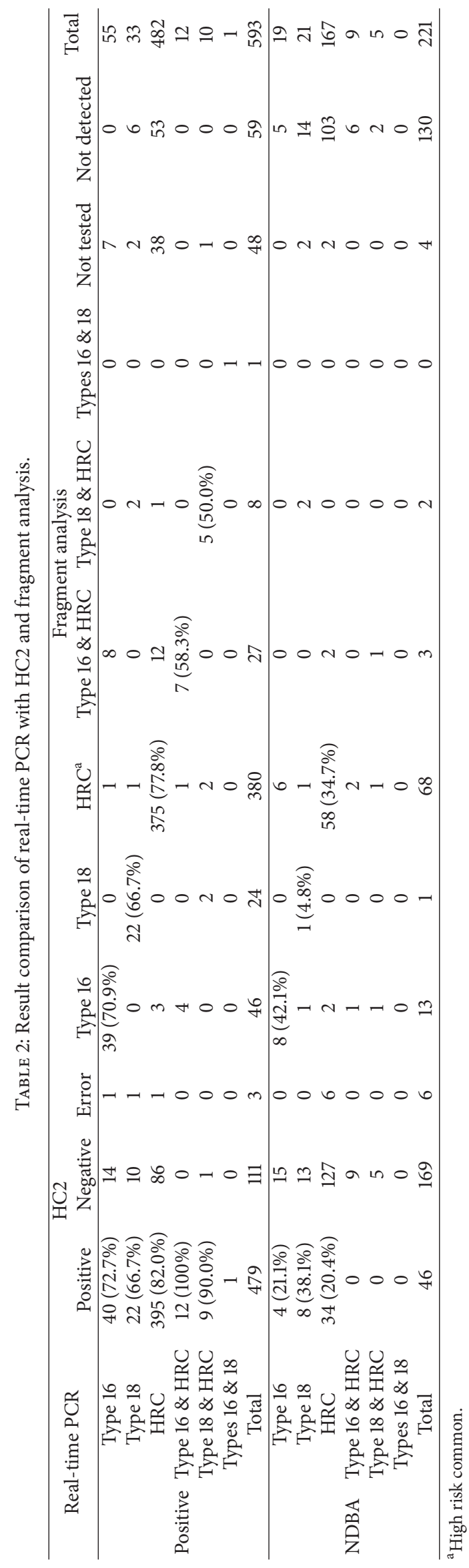


TABLE 3: Result comparison of fragment analysis with real-time PCR and HC2 in positive results with real-time PCR.

\begin{tabular}{|c|c|c|c|c|c|c|c|c|c|c|}
\hline \multirow{2}{*}{$\begin{array}{l}\text { Fragment } \\
\text { analysis }\end{array}$} & \multicolumn{6}{|c|}{ Real-time PCR } & \multicolumn{3}{|c|}{$\mathrm{HC} 2$} & \multirow{2}{*}{ Total } \\
\hline & Type 16 & Type 18 & $\mathrm{HRC}^{\mathrm{a}}$ & Type 16 \& HRC & Type 18 \& HRC & Types $16 \& 18$ & Positive & Negative & Error & \\
\hline Type 16 & $39(84.8 \%)$ & 0 & 3 & 4 & 0 & 0 & $31(67.4 \%)$ & 14 & 1 & 46 \\
\hline Type 18 & 0 & $22(91.7 \%)$ & 0 & 0 & 2 & 0 & $17(70.8 \%)$ & 6 & 1 & 24 \\
\hline HRC & 1 & 1 & 375 (98.7\%) & 1 & 2 & 0 & $331(87.1 \%)$ & 48 & 1 & 380 \\
\hline $\begin{array}{l}\text { Type } 16 \& \\
\text { HRC }\end{array}$ & 8 & 0 & 12 & $7(25.9 \%)$ & 0 & 0 & $22(81.4 \%)$ & 5 & 0 & 27 \\
\hline $\begin{array}{l}\text { Type } 18 \text { \& } \\
\text { HRC }\end{array}$ & 0 & 2 & 1 & 0 & $5(62.5 \%)$ & 0 & $7(87.5 \%)$ & 1 & 0 & 8 \\
\hline $\begin{array}{l}\text { Types } 16 \& \\
18\end{array}$ & 0 & 0 & 0 & 0 & 0 & 1 & 1 & 0 & 0 & 1 \\
\hline Total & 48 & 25 & 391 & 12 & 9 & 1 & 409 & 74 & 3 & 486 \\
\hline
\end{tabular}

${ }^{a}$ High risk common.

TABLE 4: Result comparison of fragment analysis with real-time PCR and HC2 in NDBA results with real-time PCR.

\begin{tabular}{|c|c|c|c|c|c|c|c|c|c|c|}
\hline \multirow{2}{*}{$\begin{array}{l}\text { Fragment } \\
\text { analysis }\end{array}$} & \multicolumn{6}{|c|}{ Real-time PCR } & \multicolumn{3}{|c|}{$\mathrm{HC} 2$} & \multirow{2}{*}{ Total } \\
\hline & Type 16 & Type 18 & $\mathrm{HRC}^{\mathrm{a}}$ & Type 16 \& HRC & Type 18 \& HRC & Types 16 \& 18 & Positive & Negative & Error & \\
\hline Type 16 & $8(61.5 \%)$ & 1 & 2 & 1 & 1 & 0 & $3(23.1 \%)$ & 9 & 1 & 13 \\
\hline Type 18 & 0 & 1 & 0 & 0 & 0 & 0 & 0 & 1 & 0 & 1 \\
\hline HRC & 6 & 2 & $58(84.1 \%)$ & 2 & 1 & 0 & $21(30.4 \%)$ & 44 & 4 & 69 \\
\hline $\begin{array}{l}\text { Type } 16 \& \\
\text { HRC }\end{array}$ & 0 & 0 & 2 & 0 & 1 & 0 & 0 & 3 & 0 & 3 \\
\hline $\begin{array}{l}\text { Type } 18 \& \\
\text { HRC }\end{array}$ & 0 & 1 & 0 & 0 & 0 & 0 & 0 & 1 & 0 & 1 \\
\hline $\begin{array}{l}\text { Types } 16 \& \\
18\end{array}$ & 0 & 0 & 0 & 0 & 0 & 0 & 0 & 0 & 0 & 0 \\
\hline Total & 14 & 5 & 62 & 3 & 3 & 0 & 24 & 58 & 5 & 87 \\
\hline
\end{tabular}

${ }^{\mathrm{a}}$ High risk common.

TABLE 5: Comparison of four cut-off $C_{T}$ values in real-time PCR to fragment analysis for detection of high risk HPV $(n=763)$.

\begin{tabular}{|c|c|c|c|c|c|c|c|}
\hline \multirow{2}{*}{ HPV type } & \multirow{2}{*}{ Cut-off $C_{T}$} & \multicolumn{4}{|c|}{ Number of specimens with results } & \multirow{2}{*}{$\%$ Sensitivity } & \multirow{2}{*}{$\%$ Specificity } \\
\hline & & $\mathrm{TP}^{\mathrm{b}}$ & $\mathrm{FP}^{\mathrm{c}}$ & $\mathrm{TN}^{\mathrm{d}}$ & $\mathrm{FN}^{\mathrm{e}}$ & & \\
\hline \multirow{4}{*}{16} & 31 & 54 & 2 & 671 & 36 & 60.0 & 99.7 \\
\hline & 32 & 59 & 2 & 671 & 31 & 65.6 & 99.7 \\
\hline & 33 & 62 & 2 & 671 & 28 & 68.9 & 99.7 \\
\hline & 34 & 65 & 6 & 667 & 25 & 72.2 & 99.1 \\
\hline \multirow{4}{*}{18} & 31 & 31 & 7 & 721 & 4 & 88.6 & 99.0 \\
\hline & 32 & 32 & 9 & 713 & 3 & 91.4 & 98.8 \\
\hline & 33 & 33 & 10 & 718 & 2 & 94.3 & 98.6 \\
\hline & 34 & 33 & 12 & 716 & 2 & 94.3 & 98.4 \\
\hline \multirow{4}{*}{$\mathrm{HRC}^{\mathrm{a}}$} & 31 & 369 & 49 & 218 & 127 & 74.4 & 81.6 \\
\hline & 32 & 403 & 62 & 205 & 93 & 81.3 & 76.8 \\
\hline & 33 & 424 & 81 & 186 & 72 & 85.5 & 69.7 \\
\hline & 34 & 447 & 107 & 160 & 49 & 90.1 & 59.9 \\
\hline
\end{tabular}

\footnotetext{
${ }^{\mathrm{a}}$ High risk common.

${ }^{b}$ True positive.

${ }^{\mathrm{c}}$ False positive.

$\mathrm{d}_{\text {True negative. }}$

${ }^{\mathrm{e}}$ False negative.
} 


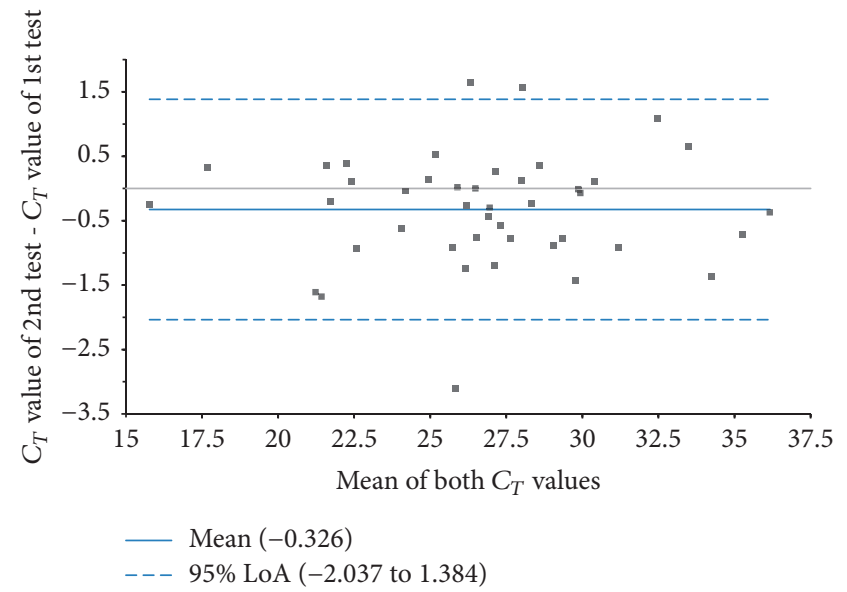

(a)

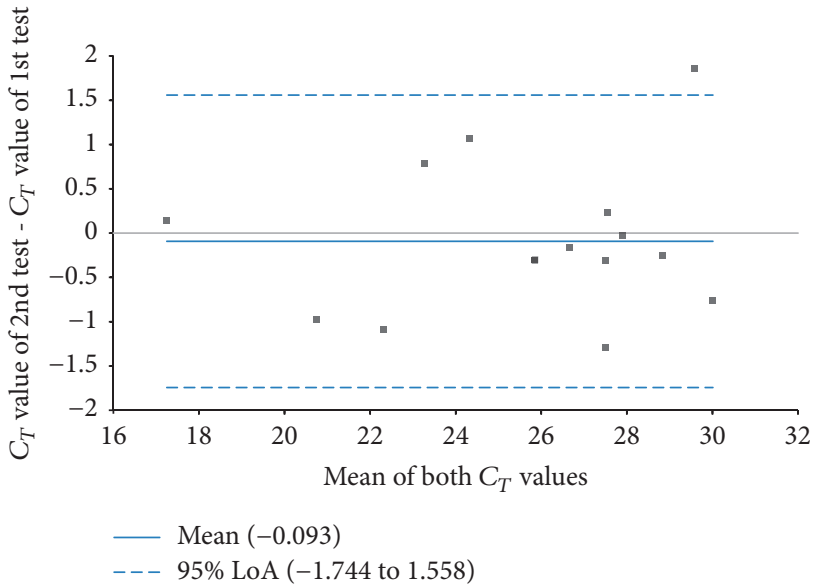

(b)

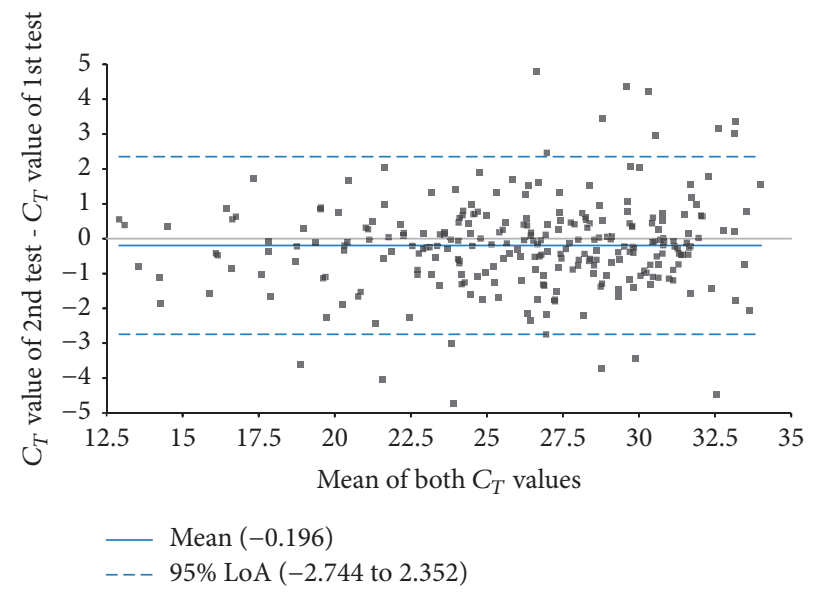

(c)

FIgURE 1: Difference plot in real-time PCR results for detection of specific HPV type. (a) Type 16: 43 specimens. (b) Type 18: 15 specimens. (c) High risk common: 265 specimens.

number per cell [19]. Integration seems to be an important event in the series of events leading to the development of cervical cancer $[19,20]$. Alternatively, low level persistence may signify containment of HPV-infected cells by cellular immunity resulting in a small lesion that may be difficult to adequately sample by standard methods. Therefore, the clinical significance of low level persistent infection of HPV and the appropriate interpretation of low level HPV DNA existence become more important [21].

\section{Conclusions}

Other reports specifying NDBA do not exist at our level of knowledge. But there is a possibility that the NDBA is expected to be described as "undetected" in the published papers. According to a retrospective study, there were 14 (2.2\%) undetected cases in 635 CIN III cases using HC2 [22]. In addition, as around $50 \%$ of ASC-US specimens will be tested high risk HPV positive, the accurate early detection of relevant infections by noninvasive and cost-effective tests is thought to be fundamental [23].

To figure out the accurate infection status of HPV (new infection, reinfection, or persistent infection), NDBA results by real-time PCR should not be overlooked and regarded as equivocal, not negative. Additionally, the adoption of different cut-off $C_{T}$ value is recommended for each high risk HPV type. Large-scale research can be needed to be backed up, as HPV infection may disappear on its own in longterm follow-up through the actions of the immune system, may remain just persistent, or contribute to the occurrence of the cancerous lesions in some patients. Psychosocial stresses from further work up can be minimized through the counselling of physicians. Virtually, all cases of cervical cancer are caused by HPV and no test will be perfect $[24]$.

\section{Ethical Approval}

This study was reviewed and approved by the Seoul National University Bundang Hospital Institutional Review Board (IRB nos. B-1207/164-304 and B-1501-284-116).

\section{Competing Interests}

The authors declare that there is no conflict of interests. 


\section{Acknowledgments}

This study is funded by Seoul National University Bundang Hospital Research Fund (Grants nos. 11-2012-011 and 02-2013068).

\section{References}

[1] G. Y. F. Ho, R. Bierman, L. Beardsley, C. J. Chang, and R. D. Burk, "Natural history of cervicovaginal papillomavirus infection in young women," The New England Journal of Medicine, vol. 338, no. 7, pp. 423-428, 1998.

[2] N. F. Schlecht, S. Kulaga, J. Robitaille et al., "Persistent human papillomavirus infection as a predictor of cervical intraepithelial neoplasia," Journal of the American Medical Association, vol. 286, no. 24, pp. 3106-3114, 2001.

[3] L. A. Koutsky, K. K. Holmes, C. W. Critchlow et al., "A cohort study of the risk of cervical intraepithelial neoplasia grade 2 or 3 in relation to papillomavirus infection," The New England Journal of Medicine, vol. 327, no. 18, pp. 1272-1278, 1992.

[4] M. Arbyn, X. Castellsagué, S. de sanjosé et al., "Worldwide burden of cervical cancer in 2008," Annals of Oncology, vol. 22, no. 12, pp. 2675-2686, 2011.

[5] Z. Zuo, S. Goel, and J. E. Carter, "Association of cervical cytology and HPV DNA status during pregnancy with placental abnormalities and preterm birth," American Journal of Clinical Pathology, vol. 136, no. 2, pp. 260-265, 2011.

[6] M. F. Evans, C. S.-C. Adamson, L. M. Schned et al., "HPV is detectable in virtually all abnormal cervical cytology samples after reinvestigation of HPV negatives with multiple alternative PCR tests," Diagnostic Molecular Pathology, vol. 19, no. 3, pp. 144-150, 2010.

[7] H. C. Kitchener, C. Gilham, A. Sargent et al., "A comparison of HPV DNA testing and liquid based cytology over three rounds of primary cervical screening: extended follow up in the ARTISTIC trial," European Journal of Cancer, vol. 47, no. 6, pp. 864-871, 2011.

[8] E.-M. De Villiers, C. Fauquet, T. R. Broker, H.-U. Bernard, and H. Zur Hausen, "Classification of papillomaviruses," Virology, vol. 324, no. 1, pp. 17-27, 2004.

[9] N. Muñoz, F. X. Bosch, S. de Sanjosé et al., "Epidemiologic classification of human papillomavirus types associated with cervical cancer," The New England Journal of Medicine, vol. 348, no. 6, pp. 518-527, 2003.

[10] A.-B. Moscicki, L. Widdice, Y. Ma et al., "Comparison of natural histories of human papillomavirus detected by clinician-and self-sampling," International Journal of Cancer, vol. 127, no. 8, pp. 1882-1892, 2010.

[11] S. de Sanjosé, M. Diaz, X. Castellsagué et al., "Worldwide prevalence and genotype distribution of cervical human papillomavirus DNA in women with normal cytology: a metaanalysis," Lancet Infectious Diseases, vol. 7, no. 7, pp. 453-459, 2007.

[12] D. Solomon, D. Davey, R. Kurman et al., "The 2001 bethesda system: terminology for reporting results of cervical cytology," Journal of the American Medical Association, vol. 287, no. 16, pp. 2114-2119, 2002.

[13] M. Poljak, A. Oštrbenk, K. Seme et al., "Comparison of clinical and analytical performance of the Abbott RealTime High Risk HPV test to the performance of hybrid capture 2 in population-based cervical cancer screening," Journal of Clinical Microbiology, vol. 49, no. 5, pp. 1721-1729, 2011.
[14] S.-M. Kulmala, S. Syrjänen, I. Shabalova et al., "Human papillomavirus testing with the hybrid capture 2 assay and PCR as screening tools," Journal of Clinical Microbiology, vol. 42, no. 6, pp. 2470-2475, 2004.

[15] S. Monnier-Benoit, V. Dalstein, D. Riethmuller, N. Lalaoui, C. Mougin, and J. L. Prétet, "Dynamics of HPV16 DNA load reflect the natural history of cervical HPV-associated lesions," Journal of Clinical Virology, vol. 35, no. 3, pp. 270-277, 2006.

[16] M. Schmitt, C. Depuydt, I. Benoy et al., "Multiple human papillomavirus infections with high viral loads are associated with cervical lesions but do not differentiate grades of cervical abnormalities," Journal of Clinical Microbiology, vol. 51, no. 5, pp. 1458-1464, 2013.

[17] S. Huang, B. Erickson, N. Tang et al., "Clinical performance of Abbott RealTime High Risk HPV test for detection of highgrade cervical intraepithelial neoplasia in women with abnormal cytology," Journal of Clinical Virology, vol. 45, supplement 1, pp. S19-S23, 2009.

[18] A. Molijn, B. Kleter, W. Quint, and L.-J. Van Doorn, "Molecular diagnosis of human papillomavirus (HPV) infections," Journal of Clinical Virology, vol. 32, supplement 1, pp. S43-S51, 2005.

[19] S. I. Collins, C. Constandinou-Williams, K. Wen et al., "Disruption of the E2 gene is a common and early event In the natural history of cervical human papillomavirus infection: a longitudinal cohort study," Cancer Research, vol. 69, no. 9, pp. 3828-3832, 2009.

[20] W. Li, W. Wang, M. Si et al., "The physical state of HPV16 infection and its clinical significance in cancer precursor lesion and cervical carcinoma," Journal of Cancer Research and Clinical Oncology, vol. 134, no. 12, pp. 1355-1361, 2008.

[21] B. Weaver, M. Shew, B. Qadadri et al., "Low-level persistence of human papillomavirus 16 DNA in a cohort of closely followed adolescent women," Journal of Medical Virology, vol. 83, no. 8, pp. 1362-1369, 2011.

[22] M. Motamedi, G. Böhmer, H. H. Neumann, and R. von Wasielewski, "CIN III lesions and regression: retrospective analysis of 635 cases," BMC Infectious Diseases, vol. 15, no. 1, article 541, 2015.

[23] M. F. Evans, C. S.-C. Adamson, J. L. Papilio, T. L. S. John, G. Leiman, and K. Cooper, "Distribution of human papillomavirus types in ThinPrep papanicolaou tests classified according to the Bethesda 2001 terminology and correlations with patient age and biopsy outcomes," Cancer, vol. 106, no. 5, pp. 1054-1064, 2006.

[24] W. Kinney, M. H. Stoler, and P. E. Castle, "Patient safety and the next generation of HPV DNA tests," American Journal of Clinical Pathology, vol. 134, no. 2, pp. 193-199, 2010. 

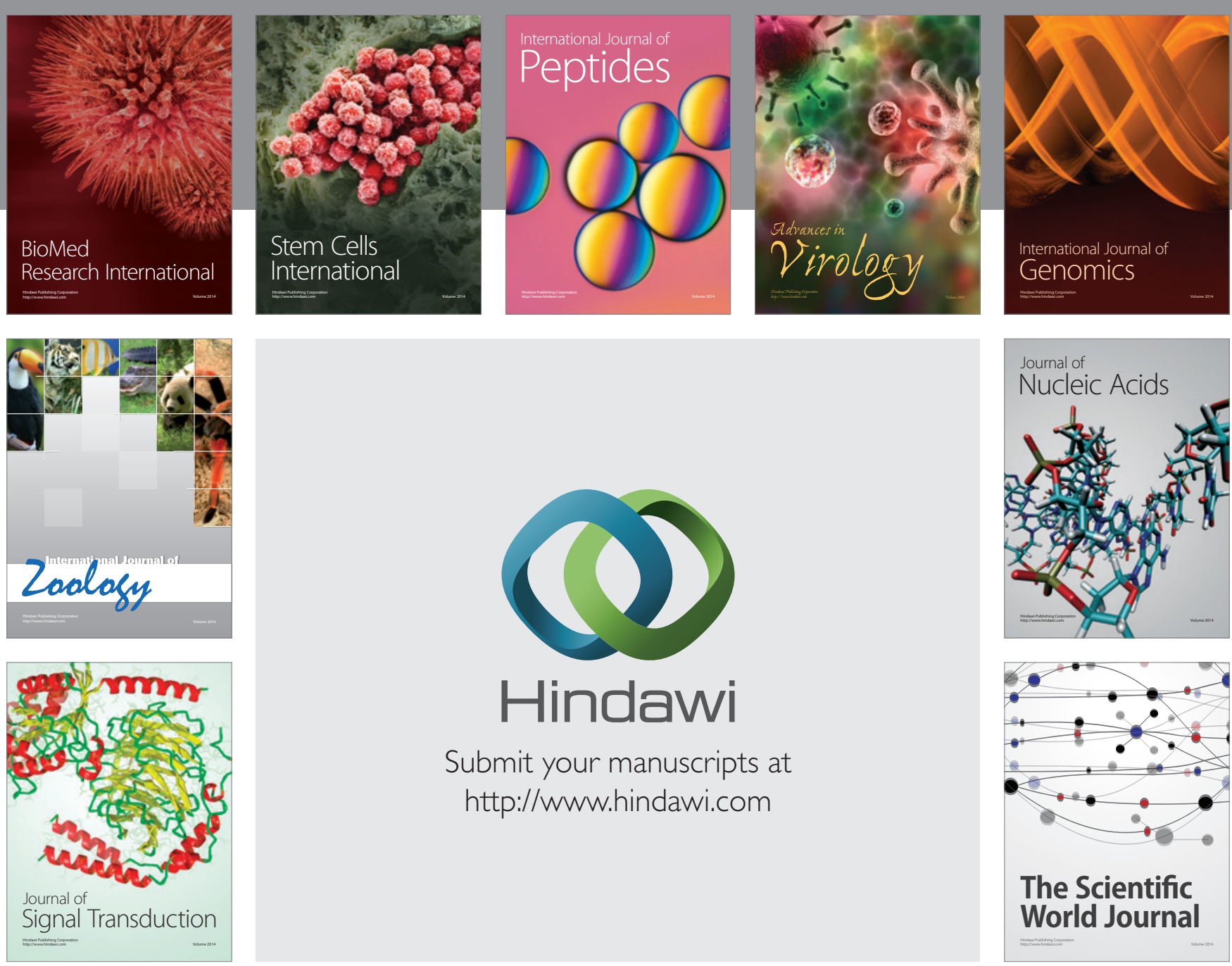

Submit your manuscripts at

http://www.hindawi.com
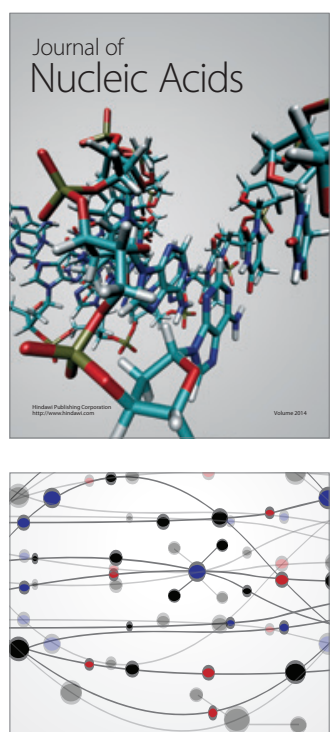

The Scientific World Journal
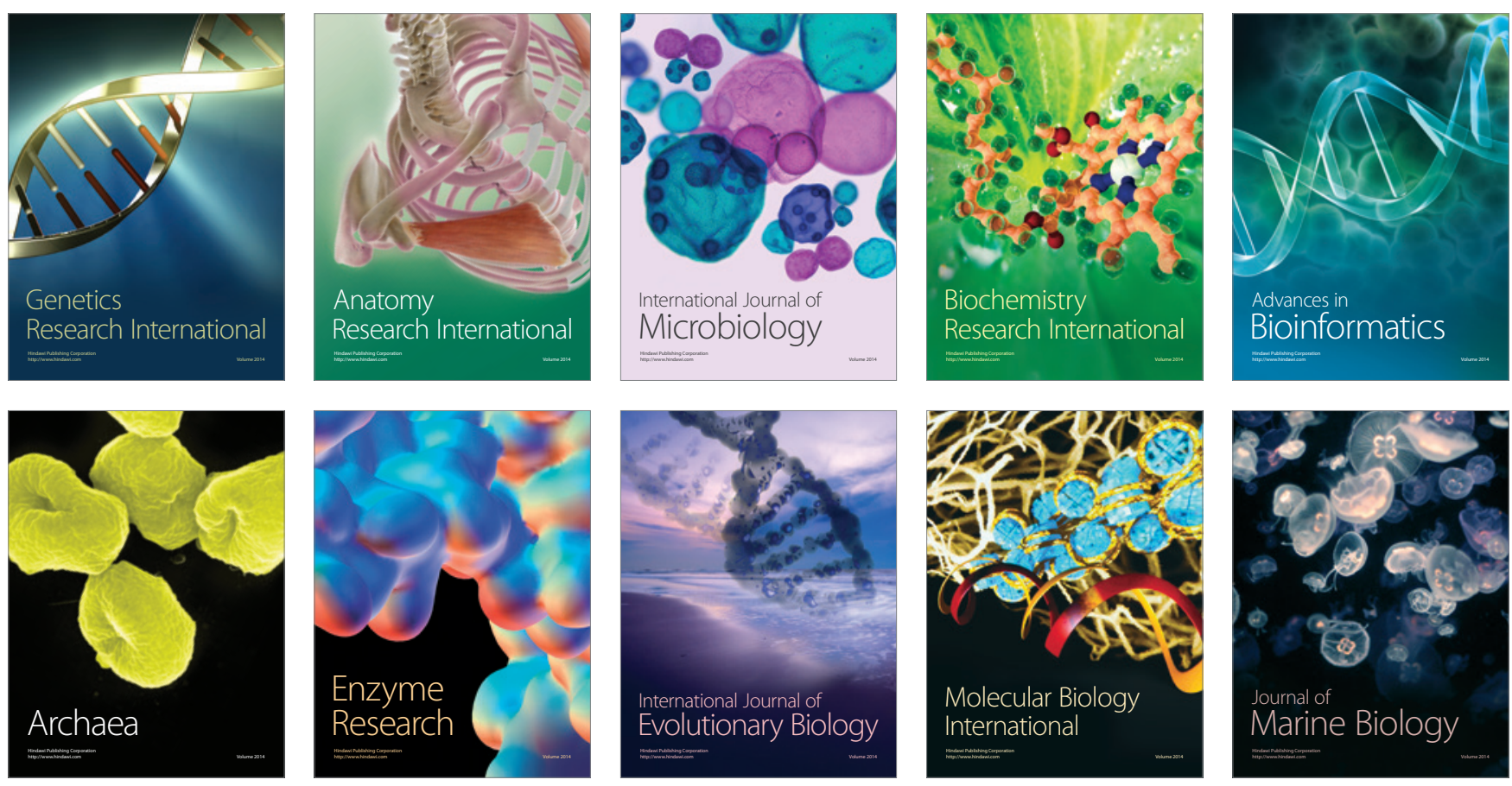